ISSN:2321-1098

\title{
Quality of Life and Nutrition During Pregnancy
}

\author{
Mitra Mirsanjari1*, Wan Abdul Manan Wan Muda1, Affizal Ahmad1 and \\ Mohd shukri Othman2 \\ 1School of Health Science, PPSK, Universiti Sains Malaysia, Kubang Kerian, \\ Kelantan, Malaysia \\ 2School of Medical Science, PPSP, Universiti Sains Malaysia, Kubang Kerian, \\ Kelantan, Malaysia
}

\begin{abstract}
A community's transition towards modernity and urbanization leads to lifestyle changes that influence an individual's health through modifications in physical activity and nutritional habits. Women of childbearing age, particularly during pregnancy require special attention with regards to nutrition because their health potentially affects the well being of the entire community beside herself and the fetus. The aim of this study was to explore the association between quality of life and the nutrition knowledge and dietary intake among a group of pregnant women in Malaysia. A cross sectional survey was carried out to explore the association of knowledge, attitudes and healthy nutritional practices with quality of life during the second and third trimester of pregnancy. In this study, nutritional habits during pregnancy were significantly associated with social functioning, vitality and physical functioning. A basic understanding of the influence a healthy lifestyle has on physical and emotional well being during pregnancy helps the mother and family members to have a positive attitude towards the need for healthy behaviors in regard to nutrition and physical activity. The findings of this study show the importance of nutritional intake and behavior with respect to their effects on dimensions of health quality.
\end{abstract}

\section{Indexing terms/Keywords}

Nutritional Behaviors, Pregnancy, Quality of Life

\section{Academic Discipline And Sub-Disciplines}

Nutrition; Nutrition in Pregnancy

\section{Subject classification}

Maternal Nutrition

\section{Type (method/approach)}

Cross Sectional Study

\section{Council for Innovative Research}

Peer Review Research Publishing System

Journal of Social Science Research

Vol.4, No.1

editor@jssronline.com 


\section{www.cirworld.com, www.jssronline.com}

\section{INTRODUCTION}

Pregnancy is generally viewed as a common and positive event [1], although it may sometimes negatively affect a woman's quality of life and her sense of well-being [2,3]. It is documented that nutritional intake is essential in achieving not only the proper growth and development of the fetus and placenta [4], but also an uncomplicated delivery, desirable maternal health and well-being [5]. During normal pregnancy, physical functioning and perception of well-being have been shown to decline as pregnancy progresses compared with pre-pregnancy [6,7]. Studies on the quality of life during pregnancy have revealed its association with various factors, such as conceptualization of optimism/pessimism [8], nausea and vomiting [9], unintended pregnancy [10], mental disorders, gestational diabetes, rheumatic diseases [11], depression, social support, and sleep [12]. However, little evidence has shown the impact of nutrient intake on maternal well-being and quality of life profiles worldwide.

To date, insufficient studies have been conducted on the association between antenatal nutrition and dietary intake with maternal physical and mental health among Malay pregnant women. Most studies in Malaysia were conducted to explore quality of life under various disease conditions. Azlin and Rizal (2009) [13] compared the quality of life of stroke survivors with that of the general Malaysian population. The impact of various demographic characteristics on quality of life profiles of Malaysian epilepsy patients was studied by Lua, Haron, Cosmas, and Nawi (2007) [14]. In another study [15], researchers explored the prevalence of anxiety and depressive symptoms, as well as their association with health-related quality of life profiles. Meanwhile, Affizal and Samaneh (2012) [16] have identified the consequences of chronic pain on patient's quality of life. The chronic patient claimed that pain prevented them from performing their daily activities effectively and efficiently.

This study aims to reveal the association between maternal nutrition and subdomains of a mother's quality of life as well as the relationship between nutrition intake and quality of life during pregnancy. The findings of this part of the study highlight the importance of good dietary habits toward achieving physical and mental health as an important determinant for the successful maternal health and pregnancy outcomes.

\section{METHODOLOGY}

\subsection{Study Location}

Data collection was performed at the Obstetrics and Gynecology Antenatal Clinic in Hospital Universiti Sains Malaysia during routine antenatal check-up for pregnant women. Obstetricians in the antenatal clinic were aware of the study, and they have communicated with the research team when a pregnant woman participates in the study.

\subsection{Study Design}

A sample of 401 pregnant women was selected systematically to participate in the study on three specified days per week, over a 13-month period. Systematic sampling of women was conducted following the numbers registered on that day. Demographic and medical history data were collected 
from the recorded information, self-administered questionnaires, and interviews. Following the confinement, the data about the pregnancy outcome were documented from the participant's folder.

The protocols of the study were evaluated and approved by the Institutional Review Board of the Health Sciences Campus and Human Research Ethical Committee of the Universiti Sains Malaysia project code [JEPeM 229.4.(1.10)].

\subsection{Selection Criteria Of Participants}

Careful selection of samples is important to ensure that the sample selected will be representative of the population to be studied. The following criteria have been used to determine the sample to be included and excluded from the study.

\section{(A) Inclusion Criteria}

(i). Gestational age between the 20th and 34th weeks from the time of the last menstrual period. This definition of gestational age was preferred so that the discomforts caused by nausea or vomiting in the first trimester had already occurred and those that are related to late pregnancy, such as backache, lower limb edema, or breathing difficulties are not yet observed [17].

(ii). Planned for vaginal delivery.

(iii). A low-risk pregnancy or pregnancy free from complications.

(iv). Malay ethnicity.

(v). Single fetus.

(vi).The range of typical childbearing age was indicated to be from 18 to 44 years [12].

\section{(B) Exclusion Criteria}

(i). Diagnosed with high-risk pregnancy at the time of enrollment, which includes conditions such as a history of diabetes mellitus, hyper/hypothyroidism, cervical incompetence, any gastric disorders, chronic hypertension, pre-eclampsia, placenta previa, and bronchial asthma.

(ii). Unable to communicate with research staff (e.g., language or mental state).

(iii). Gestational age below 20 weeks or above 34 weeks.

(iv). Medical or physical condition that prohibits regular daily activity.

(v). Multiple fetus.

(vi). Women infected with blood-borne diseases, such as HIV or syphilis were also excluded from the study, as well as women under any psychotropic medication or women who have a psychotic illness.

\subsection{Recruitment}

During the antenatal care visits, the women who have met the criteria received an explanation about the procedure of the study, and verbal consent was obtained initially. After submitting the written informed consent, the women were given an anonymous three-part questionnaire to complete. The study involved a three-step process. The first step was extracting some information from the patients' folders. In the second step, the participants complete a set of self-administered questionnaire while waiting for the physician visits. The third step involved a fifteen-minute interview for 24 diet recall, which was performed by the research member during the antenatal booking. 


\subsection{Sample Size}

Single mean formula and G power were used to calculate the sample size for the determination of variable scores, but a larger number was selected as a final sample size. Calculation of sample size showed that a total of 401 participants were needed for this study, according to the $95 \%$ confidence interval.

\subsection{Instruments}

\subsubsection{KAP Questionnaire}

The participants' nutritional knowledge, attitude, and practices were assessed using a validated questionnaire developed by the Technical Working Group on Research (TWG-R) [18]. The KAP questionnaire contained 56 questions, including 15 demographic questions; 21 item of knowledge, 8 for attitude, and 12 practice questions with 3 and 8 subdivisions for the last two questions, respectively. The knowledge questions signified five components (i.e., nutrient function, energy of food, nutrient insufficiency, food selection, and supplies of nutrients), whereas the attitude and practice questions represent four concepts (i.e., dietary intake, role of food on health, food alternative, and quality of diet). The questionnaire was tested for understandability and clarity by some students and staff of the school before the actual data collection. In calculating the nutrition knowledge scores, one mark was given to every correct response, whereas no mark to an incorrect or unsure response. The lowest possible score was zero, whereas the highest was 21.

\subsubsection{4-Hour Dietary Recalls}

The data on nutrient and dietary group intake were obtained through a single 24-hour dietary recall interview. This method is appropriate in measuring the current nutrient intake in dietary crosssectional studies $[19,20]$. The average amount of the following items were evaluated: water, energy, carbohydrate, protein, fat, fiber, calcium, phosphorous, iron, sodium, potassium, magnesium, carotene, retinol, folate, $\mathrm{RE}$, zinc, copper, selenium, omega 3 and omega 6 , as well as vitamins $A, D, E, C, B 1, B 2, B 3, B 5, B 6$, and B12. These values were then compared with the recommended nutrient intake $(\mathrm{RNI})$, or recommended dietary allowance (RDA) and estimated average requirement (EAR) during pregnancy for Malaysian population. Throughout data entry, if a food item mentioned by the participant was not originally included in the database, a similar meal from the list was replaced for assessment.

\subsubsection{SF-36 Questionnaire}

In the current study, the validated Malay version of SF-36 health survey was used to measure health status of the quality of life. This instrument was verified as a common and useful scale in general population surveys on various health conditions [1]. This questionnaire contained 36 items that were scored in the following eight domains: perception of general health (five questions), role limitations because of physical functioning (ten items), bodily pain (two items), mental health (five items), role limitations as a result of emotional problems (three items), vitality (four items), and social functioning (two items). After recording the answers to new scores and summing each domain, a formula was used to transform each domain into a score, from 0 to 100 , which represent the percentage of each domain. Therefore, a final score for each domain in the SF-36 was changed into a score of 0 to 100 , which indicate the percentage of the achieved score, whereas a score of 100 indicates optimal well-being [17,7]. 


\section{ISSN:2321-1098}

Norm-based comparison is the key to determining whether a group or an individual achieves a score below or above the average for their country, age, or sex [21]. Scores are considered significantly different from normative populations if the means differ by $>1 \mathrm{SD}$. Although scores below the mean of the general population can be interpreted as below average, statistical significance can be reached when such difference is greater than the SD for the mean of the normative population [22].

\section{STATISTIC ANALYSIS OF THE MAIN STUDY}

The data were analyzed using the Statistical Package for Social Science (SPSS) version 18. Prior to the main analysis, the data were screened for accuracy, missing values, outliers, and basic assumption. Results of continuous variables were presented as means and standard deviations, while categorical data were expressed as percentage and frequency. Mean differences of two and more different groups were tested by independent $t$-test or Mann Whitney $U$ statistics and one-way ANOVA. Differences were considered significant if their $p$ values were less than .05 . The multiple linear regressions were used to describe the relationship of the independent variables to the dependent variables. Differences were considered significant if their $p$ values were less than .05 .

\section{RESULTS}

The mean age was 29.7 years $(S D=5.04$, range was 18 to 46 years). More than half of the participants were employed $(76.5 \%)$ at the time of enrolment and they have had university education (60.6\%). The monthly family income in Ringgit Malaysia (RM) was grouped into three categories: less than or equal to RM 1500 (28.9\%); RM 1501-3500 (39.2\%); and more than RM $3500(31.4 \%)$. Nearly half of participants spent less than RM 500 of their salary for monthly food expenses of family. Pregnant women were in the second or third trimester of pregnancy with a frequency of $54.9 \%$ and $49.1 \%$, respectively. The majority of them have not had a previous abortion $(74.8 \%)$ and were multigravida $(59.1 \%)$ with $0-1$ parity $(55.1 \%)$.

The results also showed that the participants had average score of $13.85( \pm 3.44)$ for nutrition knowledge. The frequency of good, moderate and poor nutrition knowledge were $52.4 \%, 42.4 \%$, and $5 \%$, respectively. The majority of subjects (84\%) ate breakfast every morning, but $3.7 \%$ mentioned only having breakfast once per week or less. The reasons for avoiding breakfast included "no appetite" or "not having enough time for eating breakfast." Nearly half chose a starchy and fatty breakfast consisting of fried noodles and similar foods, mixed rice/chicken rice, while onefifth $(20 \%)$ had bread, biscuits, cookies or cereals for breakfast. About a third $(34.3 \%)$ snacked once or more a day, mainly on local cakes (kuih muih) and biscuits. Rice was the basic food chosen by $84.3 \%$, while $33.4 \%$ took rice for lunch and for dinner. Rice is usually consumed with fish, poultry, and vegetables. The majority of subjects maintained a three-meal pattern of heavy breakfast, lunch, and dinner. Fruit and vegetable were also taken at breakfast, lunch, and dinner by $0.2 \%, 0.5 \%$ and $8.2 \%$, respectively. The demographic and gestational characteristics of the sample are summarized in Table 4.1. 


\section{ISSN:2321-1098}

Table 4.1 Demographic and Obstetric Characteristics of Pregnant Women

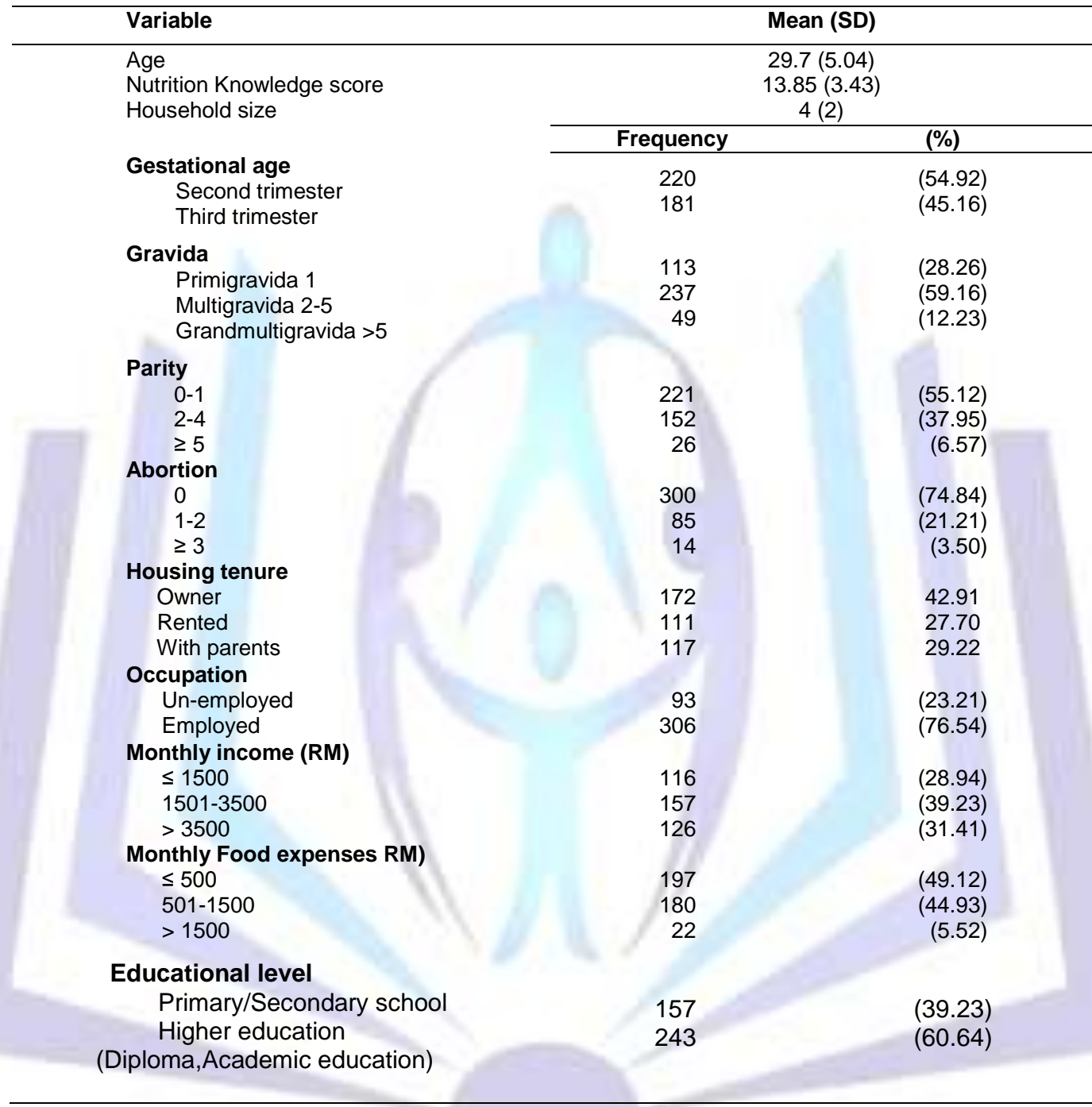

The final model of multiple linear regressions showed that occupation, education, and monthly income were positive significant demographic predictor factors for nutritional knowledge of this group of participants. Individuals with occupation have shown higher nutrition knowledge score of 1.286. Those with higher education level had 2.562 higher score of nutrition knowledge, and group of women with earnings between RM 1501- 3500, and more than RM 3500 had higher knowledge score of 1.488 and 1.989 , respectively (Table 4.2 ). 
Table 4.2- Predictor Factors for Nutrition Knowledge of Pregnant Women - Stepwise method $(n=401)$

\begin{tabular}{|c|c|c|c|c|}
\hline \multirow{2}{*}{ Variables } & \multicolumn{4}{|c|}{ Multiple Linear Regression $^{\text {b }}$} \\
\hline & Adjusted b (95\% Cl) & t statistics & $\mathbf{R}^{2}$ & p value \\
\hline Occupation & $1.286(.559,2.013)$ & 3.479 & & .001 \\
\hline $\begin{array}{l}\text { Education } \\
\text { Monthly income (RM) }\end{array}$ & $2.562(1.890,3.234)$ & 7.495 & .397 & .000 \\
\hline $1501-3500$ & $1.488(.731,2.245)$ & 3.866 & & .000 \\
\hline$>3500$ & $1.989(1.152,2.826)$ & 4.671 & & .000 \\
\hline
\end{tabular}

Notes. ${ }^{b}$ Multiple linear regression

This study revealed that the number of parity of pregnant women was significantly associated with the score of nutrition knowledge $(p=.005 ; 95 \% \mathrm{Cl}$ : $-.480,-.085)$. Women with higher parity had lower nutrition knowledge score. The findings of this study showed that total general health was the only quality of life domain that was significantly associated with nutrition knowledge $(P=.025 ; 95 \%$ $\mathrm{Cl}: .003, .047)$. The median of nutrient intake of pregnant women was obtained based on the recommendation of nutrient intake for Malaysian and recommended dietary allowance. The main energy sources included carbohydrates (51\%-61\%), followed by fat (24\%-28\%), and protein (16\%$17 \%)$. Average intake of maternal energy and protein were $1732.12(606.94) \mathrm{kcal} / \mathrm{d}$ and $73.9(30.2)$ $\mathrm{g} / \mathrm{d}$, respectively. Vitamins $A$ and $\mathrm{C}$, micronutrients rich in vegetables and fruits had the mean values of $1,453.6(1,354.4) \mu \mathrm{g}$ and 145.1 (105.2) $\mathrm{mg}$, respectively. Carbohydrate, protein, and fat were consumed excessively compared with the desired requirement; however, total energy does not meet the recommended value in both age and gestational age ranges. Figure 3.1 shows the percentage of nutrient intake in comparison with estimated average requirements (EAR). Intake of fibre and folate of pregnant women were severely lower than the recommended dietary allowance with percentage of $13.2 \%$ and $13 \%$ respectively.

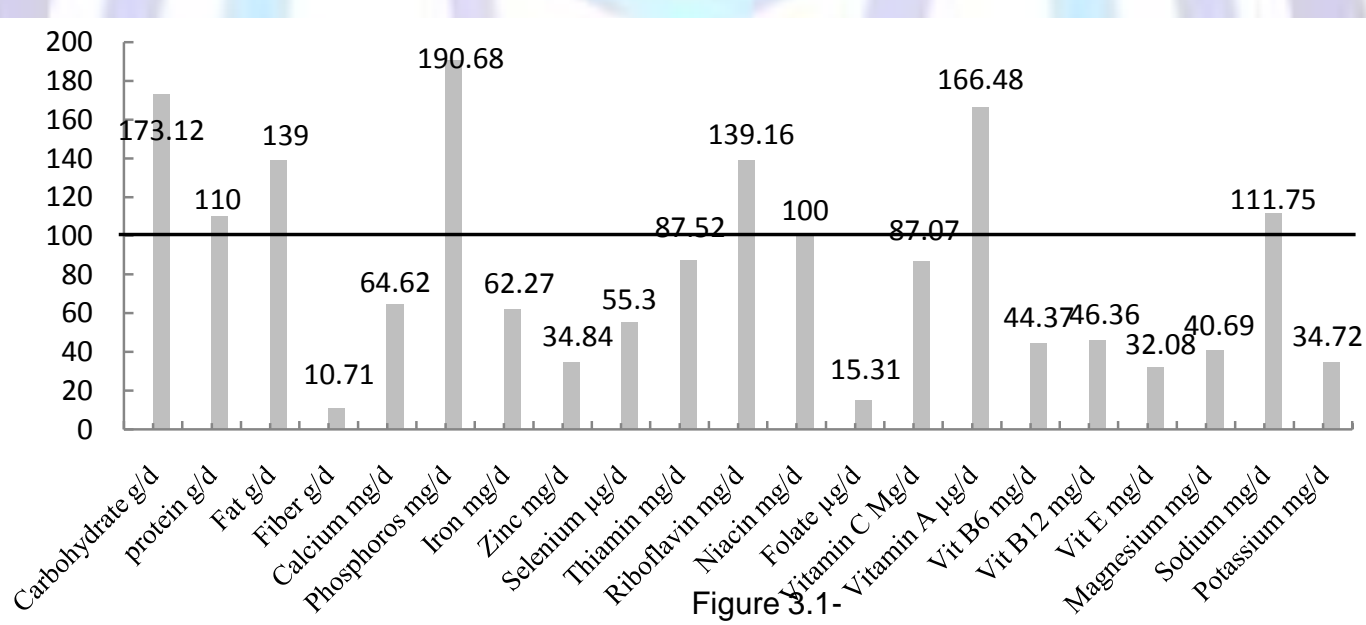

Percentage of Macronutrients and micronutrients in comparison with EAR

From the gestational determinants (gestational age, gravida, parity, abortion, craving and aversion), only gestational age was found to have a significant negative association with physical functioning score $(b=-.693 ; 95 \% \mathrm{Cl}=-1.122,-.263 ; p$ value $=.002)$. Results also showed that household size 


\section{ISSN:2321-1098}

was the only predictive demographic factor that had significant association with physical functioning level $(b=909 ; 95 \% \mathrm{Cl}=.007,1.810 ; p$ value $=.048)$. Fast food consumption was found to have significant association with physical functioning $(b=5.296 ; 95 \% \mathrm{Cl}=1.418,9.174 ; p$ value $=.008)$. Persons who rarely consumed fast food (once per month) had higher scores of physical functioning compared to those who ate more frequently. Consumption of breakfast 2-3/ month was the only predictor factor of bodily pain. Those who used to consume breakfast 2-3 times per month had lower score (more bodily pain) compared to those who had breakfast more frequently $(b=-23.172$; 95\% IC: $-39.394,-6.949$; $p$ value $=.005)$. General health of pregnant women in this study was significantly associated with educational level $(b=3.958 ; 95 \%$ IC: $.907,7.008 ; p$ value $=.011)$

Consumption of bean once per week $(b=-5.674 ; 95 \%$ IC: $-9.511,-1.836$; $p$ value $=.004)$ and once per month ( $b=-4.084$; 95\% IC: $-7.479,-.688$; $p$ value $=.019)$ was found to have negative association with general health score. Gestational age had a negative association with vitality as a predictive factor. Pregnant women with more gestational age had lower level of vitality score $(b=-$ $.339 ; 95 \%$ IC: $-.662,-.015$; $p$ value $=.040$ ). This study revealed that the vitality of pregnant women was significantly associated with intake frequency of fast food, breakfast and performing the exercise. A positive significant association was found between never consumption of fast food and vitality ( $b=9.583 ; 95 \%$ IC: $.199,18.968 ; p$ value $=.045)$. However, a negative association was found between never intake of breakfast $(b=-12.066 ; 95 \% \mathrm{IC}:-22.307,-1.825 ; p$ value $=.021)$ and never performing of exercise $(b=-6.766 ; 95 \%$ IC: $-12.546,-.985 ; p$ value $=.022)$.

The results of the current study showed that parity was significantly associated with social functioning score. Those who had more deliveries had 1.3 higher scores of social functioning $(b=$ 1.306; 95\% IC: .233, 2.389; $p$ value $=.018$ ). Results also showed that fast food consumption once per month was significantly associated with social functioning score $(b=7.088 ; 95 \%$ IC: 1.841 , $12.334 ; \mathrm{p}$ value $=.008)$. This study revealed that the role emotion of pregnant women was significantly associated with gestational age. Those who had more gestational age had lower scores of role emotion ( $b=-.862 ; 95 \%$ IC: $-1.704,-.020 ; p$ value $=.045)$.

The role emotion level was shown to have significant association with egg intake 2-3 times per week. Individuals who used to eat egg 2-3 times per weeks had 15.15 higher scores of role emotion compared to those who consumed less frequently ( $b=15.158 ; 95 \%$ IC: $2.576,27.739 ; p$ value $=.018)$. Gravida was found to have significant association with mental health. Those with more number of pregnancies had higher scores of mental health $(b=.897 ; 95 \%$ IC: $.229,1.568 ; p$ value $=.009)$. Age was shown to have significant association with mental health scores among pregnant women $(b=.486 ; 95 \%$ IC: $.215, .757$; $p$ value $=.000)$.

\subsection{Comparison Of Nutrient Intake Of Four Groups Based On Quality}

\section{Of Life Domain Scores}

To find the relationship between nutrient intake and quality of life determinants, one-way ANOVA was used to show the mean differences of macro/ micro nutrients between the quartile groups of each domain. Post hoc analysis showed the significant difference of riboflavin mean intake between $2^{\text {nd }}$ and $3^{\text {rd }}$ quartile of role physical. 
Persons whose total role physical scores were between 50.1 and 75 had higher intake of riboflavin compared to those who had total scores between 25.1 till 50. A significant difference of magnesium mean intake was shown between quartile two and three; and between quartile two and four of pregnant women. Persons whose total role physical scores were between 25.1 till 50 had lower intake of magnesium compared to those who had total scores between 50.1 and 75 or 75.1 till 100 .

The findings of the current study showed that mean intake of sodium was significantly different between $2^{\text {nd }}$ and $3^{\text {rd }}$ quartile of bodily pain scores. Persons whose total bodily pain scores were between 25.1 till 50 had lower intake of sodium compared to those who had total scores between 50.1 and 75. A significant difference of cholesterol mean intake was shown between quartile three and four of pregnant women mental health. Persons with total mental health scores between 50.1 till 75 had higher intake of cholesterol compared to those who had total scores of 75.1 to 100.

\section{DISCUSSION}

Even though the majority of pregnant women had suitable nutrition knowledge, its influence on optimum nutrient intake and dietary practices was weak among the study population. The actual food intake of pregnant women did not meet the specific nutritional needs of pregnancy, despite the majority of subjects had moderate to high nutritional knowledge. It can be assumed that "nutrition knowledge" is a necessary but insufficient factor contributing to changes in individual food intake and behavior. Dietary intake and practices are influenced by a number of environmental and intraindividual factors, including motivations [23]. The overall findings on quality of life components in the present research indicate that although pregnancy has long been considered a time of great emotional and physical disruption that may produce a predictable effect on physical function, its effect on emotional health status might be significantly more limited. In relation to the objective of this study, researcher attempted to describe the concurrent association between socio demographic and gestational characteristics with nutrient intake and dietary changes during pregnancy and quality of life.

This study revealed that "physical function", "social function" and "vitality" were significantly associated with fast food consumption. Evidence indicates that frequent consumption of fast food has adverse health implications [24]. Pereira et al. (2005) [25] showed that a more than 15-year period of fast food consumption had a strong positive association with weight gain and insulin resistance. Based on the frequent consumption of fast foods and snacks, the fast food dietary pattern characterized by high consumption of sweets, soft drinks, hamburgers, and pizza is a risk predictor for excess maternal weight gain by providing higher intakes of energy, fat, and sucrose [26]. Uusitalo (2009) [27] reported that pregnant women who belonged to the highest quartile of the fast food dietary pattern gained $1.3 \mathrm{~kg}$ more weight on average during pregnancy (10th week to 40th week) than those who belonged to the lowest quartile [27]. Current findings show that women who frequently consumed fast food had a poorer nutrition knowledge mean score compared with those who rarely consumed fast food, although the difference was not significant. Similar to previous research [28], the current study found that socioeconomic patterns in dietary intake are related to a number of factors, including educational level, employment status, income, and cultural differences. Maternal education may affect pregnancy outcome through acquired knowledge of health care progressions or through its connection with other aspects related to healthy life style [29]. 
In the present study, a significant difference was observed in the monthly income of women who frequently consumed fast food compared with those who rarely consumed fast food. A significant association was found between the educational level of individuals and their frequency of fast food intake. The finding of the current study also showed that the bodily pain domain score was associated with consumption of breakfast. Individuals who used to consume breakfast twice or trice per month had lower score (more bodily pain) compared with those who consumed breakfast more frequently. The first meal of the day has long been considered as important to start a day with a healthy condition physiologically and psychologically. Evidence demonstrates that the macronutrient composition of breakfast is positively associated with conscientiousness, wellbeing, general health [30], decline in fatigue and dysphoria [31], alertness [32], feeling of being sociable and outgoing, and reduced discontentment [33].

In this study, the majority of respondents reported that they ate breakfast daily. This finding could be noteworthy, considering that $76.2 \%$ of the participants were either government or private employees. Skipping breakfast might be the consequence of engaging in executive or educational tasks with justification of lack of time or being too busy to prepare breakfast. Skipping breakfast has been well documented to have a prejudicial outcome on an individual's performance and mood. This association may be explained by the fact that consuming breakfast, specifically complex carbohydrates, elevates the release of a specific brain chemical, a neurotransmitter called serotonin. Serotonin induces the feeling of calm and relaxation as well as also provides immediate energy and a sense of well-being [34].

The "general health" scores of participants were significantly lower in individuals who consumed bean products once per week or once per month compared with those who consumed such products every day or 2-3 times per week. A significant, but weak correlation was found between the amount of water consumption and "vitality" component. In this study, pregnant women who had daily intake of vitamin and mineral supplements showed lower bodily pain compared with those who never used such supplements. Moreover, the "social functioning" of individuals who took supplements once per week was significantly lower than that of individuals who took supplements twice or thrice per week or more.

As regards the study objectives, predictive factors of demographic, gestational, and dietary variables were determined with quality of life components by controlling possible confounders after adjusting for other potential factors. The vitality scores of pregnant women were significantly and positively associated with lower consumption of fast food and the performance of 20 minutes of exercise daily or two to three times per week. The recent finding may be explained by the fact that exercise stimulates the release of phenylethylamine, which is the endorphin-induced chemical component that promotes attention, energy, and mood [35]. This finding confirms the results of other research in which women with optimized physical activity at approximately 20 weeks of gestation showed an improved sense of vigor and a decline in fatigue [36]. However, Tendais et al. (2011), in a study on the relationship between physical activity patterns and quality of life from prepregnancy to the second trimester of pregnancy, reported that a decrease in most physical dimensions and an increase in the mental component were independently related to physical activity status [37].

Lower vitality scores with higher consumption of fast food can be explained by the fact that consuming fast food may cause a series of detrimental effects, ranging from depression to hyperactivity. This condition results in a physical or mental malfunction owing to the brain 
imbalances caused by the toxic combination of unhealthy fats, food preservatives, artificial coloring, and refined carbohydrate fast food [38]. In this study, a significant association was found between vitality scores and amount of water intake. This recent finding can be explained by the fact that being well-hydrated is important for physical and mental health because it improves the transfer of nutrients into cells and the removal of waste from cells. The current finding confirms the results of other studies $[39,33]$, which revealed the influence of healthy dietary habits on daily performance and health. Adequate hydration during gestation provides successful pregnancy outcomes because water supports fetal growth and supports maternal physiologic adjustments [40]. Although increased water intake was shown to elevate the risk of preterm delivery and low birth weight [41], another study [42] reported its association with higher mean of fetal birth weight.

No significant association was found between health quality components and nutrient intake of individuals. However, analyses of nutrients mean differences between quartiles of quality of life were significant. Differences in mean scores were found between lower and higher quartiles of quality of life for riboflavin, sodium, magnesium, cholesterol and copper. This finding might be explained by this fact that food components significantly contribute to the antenatal quality of life in pregnant women with effects on physical functioning and occasionally on mental well-being. Adequate nutrient intake was also found to be an important determinant of health and well-being, especially during pregnancy. Good nutrition improves quality of life by promoting health, preventing dietary deficiency disease, and ameliorating or averting secondary malnutrition caused by or associated with other diseases [43]. The current study revealed the probable association between dietary practices and quality of life in a cross sectional study. However, future investigations may need to reveal this association during a longitudinal assessment, and repeated studies with consistent results can provide compelling evidence for associations.

\section{CONCLUSION}

The findings of this study revealed that majority of pregnant women had moderate or good nutritional knowledge. It seems that knowledge enhancement might cause practice modulation by affecting and changing on the attitude. However, dietary practice and daily food intake of pregnant mothers in current study does not seem to meet the recommended dietary intake. More than half of the women had inadequate energy intake and that the majority of them had inadequate intake of fiber, calcium, iron, potassium, magnesium, zinc, niacin, vitamin C, folate, vitamin B6, and vitamin B12. Occupation, education, and monthly income were significant predictor factors for nutritional knowledge in this study population. The findings of current study showed that participants' food practices were significantly associated with domains of quality of life in some cases. Physical function, social function and vitality were significantly associated with fast food consumption. The bodily pain and vitality scores of pregnant women were significantly association with consumption of breakfast. Role emotion level was shown to have significant positive association with egg intake 2-3 times per week. The vitality scores were significantly and positively associated with the performance of 20 minutes of exercise daily or two to three times per week.

\section{ACKNOWLEDGEMENTS}

We thank the pregnant women who took part in our study for being generous with their time. We appreciate the staff at the Obstetrics and Gynaecology clinic of the Hospital Universiti Sains Malaysia for their contribution to our study. We would like to acknowledge Universiti Sains Malaysia for the grant allocated to perform this study. There are no competing financial interests. 


\section{REFERENCES}

[1] Nicholson, W. K., Setse, R., Hill-Briggs, F., Cooper, L. A., Strobino, D., \& Powe, N. R. (2006). Depressive symptoms and health-related quality of life in early pregnancy. Obstetrics \& Gynecology, 107 (4), 798- 806.

[2] Hueston, W. J., \& Kasik-Miller, S. (1998). Changes in functional health status during normal pregnancy. The Journal of family practice, 47 (3), 209-212.

[3] Tasdemir, S., Balci, E., \& Gunay, O. (2010). Comparison of life quality of pregnant adolescents with that of pregnant adults in Turkey. Upsala journal of medical sciences, 115 (4), 275281.

[4] Essilfie, P. A. (2013). Evidence-Based Project: Impact of Micronutrients on Maternal Health, Child Growth and Development. International Journal of Maternal and Child Health, 1(4), 67-76.

[5] Godfrey, K. M., \& Barker, D. J. (2000). Fetal nutrition and adult disease. The American Journal of Clinical Nutrition, 71 (5), 1344s-1352s.

[6] Haas, J. S., Jackson, R. A., Fuentes-Afflick, E., Stewart, A. L., Dean, M. L., \& Brawarsky, P. (2005). Changes in the health status of women during and after pregnancy. Journal of General Internal Medicine, 20 (1), 45-51.

[7] Mckee, M. D., Cunningham, M., Jankowski, K. R., \& Zayas, L. (2001). Health-related functional status in pregnancy: relationship to depression and social support in a multi-ethnic population. Obstetrics \& Gynecology, 97 (6), 988- 993.

[8] Moyer, C. A., Yang, H., Kwawukume, Y., Gupta, A., Zhu, Y., Koranteng, I., et al. (2009). Optimism/pessimism and health-related quality of life during pregnancy across three continents: a matched cohort study in China, Ghana, and the United States. BMC pregnancy and childbirth, 9 (1), 39.

[9] Lacasse, A., \& Berard, A. (2008). Validation of the nausea and vomiting of pregnancy specific health related quality of life questionnaire. Health and quality of life outcomes, 6 (1), 32.

[10] Schwarz, E. B., Smith, R., Steinauer, J., Reeves, M. F., \& Caughey, A. B. (2008). Measuring the effects of unintended pregnancy on women's quality of life. Contraception, 78 (3), 204210.

[11] Forger, F., Oestensen, M., Schumacher, A., \& Villiger, P. M. (2005). Impact of pregnancy on health related quality of life evaluated prospectively in pregnant women with rheumatic diseases by the SF-36 health survey. Annals of the rheumatic diseases, 64 (10), 14941499.

[12] Mota, N., Cox, B. J., Enns, M. W., Calhoun, L., \& Sareen, J. (2008). The relationship between mental disorders, quality of life, and pregnancy: findings from a nationally representative sample. Journal of affective disorders, 109 (3), 300- 304.

[13] Azlin, N., \& Rizal, A. (2009). Health related quality of life (HRQOL) among stroke survivors attending rehabilitation centres in Selangor. Jurnal Kesihatan Masyarakat, 15(2), 83-90.

[14] Lua, P. L., Haron, H., Cosmas, G., \& Nawi, N. H. M. (2007). The impact of demographic characteristics on health-related quality of life profile of Malaysian epilepsy population. Applied Research in Quality of Life, 2 (4), 247- 271.

[15] Pei Lin, L., Sok Yee, W., \& Widiasmoro Selamat, N. (2011). Anxiety and depressive symptoms and health-related quality Of life status among patients with cancer in Terengganu, Malaysia. ASEAN Journal of Psychiatry, 12 (1), 1- 15. 
[16] Affizal, A., \& Samaneh, T. (2012). Understanding chronic pain patient's quality of life using Interpretative phenomenological analysis Approach. Journal of Advanced Social Research, 2 (2), 110-119.

[17] Couto, E. R., Couto, E., Vian, B., Gregorio, Z., Nomura, M. L., Zaccaria, R., et al. (2009). Quality of life, depression and anxiety among pregnant women with previous adverse pregnancy outcomes. Sao Paulo Medical Journal, 127 (4), 185-189.

[18] Karim, N. A., Safii, N. S., Yusof, S. M., Noor, N. M., Ahmad, Z., \& Tee, E. S. (2008). Nutrition knowledge among Malaysian elderly. Jurnal Sains Kesihatan Malaysia, 6 (2), 43-54.

[19] Maruyama, K., Kubota, Y., Sato, S., Ishikawa, Y., Shimamoto, T., Inagawa, M., et al. (2009). The reproducibility of 24-h dietary recall for estimating mineral intakes and their food sources among middle-aged Japanese men and women. International journal of food sciences and nutrition, 60 (s7), 30- 40.

[20] Rajeshwari, R., Nicklas, T. A., Yang, S.-J., \& Berenson, G. S. (2004). Longitudinal changes in intake and food sources of calcium from childhood to young adulthood: the bogalusa heart study. Journal of the American College of Nutrition, 23 (4), 341- 350.

[21] Azman, A., Sararaks, S., Rugayah, B., Low, L., Azian, A., Geeta, S., et al. (2003). Quality of life of the Malaysian

general population: results from a postal survey using the SF-36. Medical Journal of Malaysia, 58 (5), 694-711.

[22] Drescher, K. M., Monga, M., Williams, P., Promecene-Cook, P., \& Schneider, K. (2003). Perceived quality of life

1231-1233.

in pregnant adolescent girls. American journal of obstetrics and gynecology, 188 (5),

[23] Worsley, A. (2002). Nutrition knowledge and food consumption: can nutrition knowledge change food behaviour? Asia Pacific Journal of Clinical Nutrition, 11 (s3), S579- S585.

[24] Lhila, A. (2011). Does access to fast food lead to super-sized pregnant women and whopper babies? Economics \& Human Biology, 9 (4), 364- 380.

[25] Pereira, M. A., Kartashov, A. I., Ebbeling, C. B., Van Horn, L., Slattery, M. L., Jacobs Jr, D. R., et al. (2005). Fast-food habits, weight gain, and insulin resistance (the CARDIA study): 15year prospective analysis. The Lancet, 365 (9453), 36- 42.

[26] French, S. A., Harnack, L., \& Jeffery, R. W. (2000). Fast food restaurant use among women in the Pound of Prevention study: dietary, behavioral and demographic correlates. International journal of obesity and related metabolic disorders: journal of the International Association for the Study of Obesity, 24 (10), 1353-1359.

[27] Uusitalo, U., Arkkola, T., Ovaskainen, M.-L., Kronberg-Kippila, C., Kenward, M. G., Veijola, R., et al. (2009). Unhealthy dietary patterns are associated with weight gain during pregnancy among Finnish women. Public health nutrition, 12 (12), 2392- 2399.

[28] Dowler, E. (2001). Inequalities in diet and physical activity in Europe. Public Health Nutrition, 4 (2b), 701-709.

[29] Islam, M. M. (2013). Incidence of and Risk Factors for Low Birth Weight in Oman: A Population Based Study. International Journal of Maternal and Child Health, 1(4), 77-86.

[30] Reeves, S., Halsey, L. G., McMeel, Y., \& Huber, J. W. (2012). Breakfast habits, beliefs and measures of health and wellbeing in a nationally representative UK sample. Appetite, 60 (1), $51-57$.

[31] Lloyd, H. M., Rogers, P. J., Hedderley, D. I., \& Walker, A. F. (1996). Acute effects on mood and cognitive performance of breakfasts differing in fat and carbohydrate content. Appetite, 27 (2), 151- 164. 


\section{ISSN:2321-1098}

[32] Holt, S. (1999). The effects of high-carbohydrate vs high-fat breakfasts on feelings of fullness and alertness, and subsequent food intake. International journal of food sciences and nutrition, 50 (1), 13-28.

[33] Smith, A. P., Kendrick, A., Maben, A., \& Salmon, J. (1994). Effects of breakfast and caffeine on cognitive performance, mood and cardiovascular functioning. Appetite, 22 (1), 39- 55.

[34] Bannan, P. (2006). Eat Right When Time Is Tight: 150 Slim-Down Strategies and No-Cook Food Fixes: NorlightsPress.

[35] Szabo, A., Billett, E., \& Turner, J. (2001). Phenylethylamine, a possible link to the antidepressant effects of exercise? British Journal of Sports Medicine, 35 (5), 342- 343.

[36] Cioffi, J., Schmied, V., Dahlen, H., Mills, A., Thornton, C., Duff, M., et al. (2010). Physical activity in pregnancy: Women's perceptions, practices, and influencing factors. Journal of Midwifery \& Women's Health, 55 (5), 455-461.

[37] Tendais, I., Figueiredo, B., Mota, J., \& Conde, A. (2011a). Physical activity, health-related quality of life and depression during pregnancy. Cadernos de Saude Publica, 27 (2), 219228.

[38] Sanchez-Villegas, A., Toledo, E., de Irala, J., Ruiz-Canela, M., Pla-Vidal, J., \& MartinezGonzalez, M. A. (2012). Fast-food and commercial baked goods consumption and the risk of depression. Public Health Nutrition, 15 (3), 424- 432.

[39] Smith, A., Kendrick, A., \& Maben, A. (1992). Effects of breakfast and caffeine on performance and mood in the late morning and after lunch. Neuropsychobiology, 26 (4), 198- 204.

[40] Szwajcer, E. M., Hiddink, G. J., Koelen, M. A., \& van Woerkum, C. M. (2007). Nutrition awareness and pregnancy: Implications for the life course perspective. European Journal of Obstetrics \& Gynecology and Reproductive Biology, 135 (1), 58- 64.

[41] Heflin, C. M., Siefert, K., \& Williams, D. R. (2005). Food insufficiency and women's mental health: findings from a 3-year panel of welfare recipients. Social science \& medicine, 61 (9), 1971-1982.

[42] O'Sullivan, T. A., Robinson, M., Kendall, G. E., Miller, M., Jacoby, P., Silburn, S. R., et al. (2009). A good-quality breakfast is associated with better mental health in adolescence. Public health nutrition, 12 (2), 249- 258.

[43] Amarantos, E., Martinez, A., \& Dwyer, J. (2001). Nutrition and quality of life in older adults. The Journals of Gerontology Series A: Biological Sciences and Medical Sciences, 56 (2), 5464. 